\title{
FATORES ASSOCIADOS À OCORRÊNCIA DE EXTRAVASAMENTO EM CRIANÇAS E ADOLESCENTES DA CLÍNICA MÉDICA
}

\author{
$\underline{\text { Emily da Cruz Lima }}{ }^{1}$; Cleonara Souza Gomes e Silva ${ }^{2}$; Larissa Freitas Cerquira ${ }^{3}$; \\ Luciano Marques dos Santos ${ }^{6 .}$
}

1. Graduanda em enfermagem. Universidade Estadual de Feira de Santana, e-mail: emilylima18@ hotmail.com.

2. Graduanda em enfermagem. Universidade Estadual de Feira de Santana, e-mail: cleosilvauefs@gmail.com.

3. Graduanda em Enfermagem. Universidade Estadual de Feira de Santana, e-mail: cerqueirafreitaslarissa@gmail.com.

4. Orientador. Departamento de Saúde. Universidade Estadual de Feira de Santana, email: 1ucmarxenfo@yahoo.com.

PALAVRAS-CHAVES: Enfermagem Pediátrica; Criança hospitalizada; Segurança do Paciente; Extravasamento de materiais terapêuticos e diagnósticos.

\section{INTRODUÇÃO}

A TIV é um processo que compreende desde o preparo do paciente, a escolha e obtenção do acesso venoso periférico, o cálculo, o preparo e a administração de fármacos e soluções, as trocas de soluções, dispositivos e curativos, até a retirada dos cateteres. É indicada quando se deseja um acesso venoso para a administração de fluidos e/ou fármacos no sistema circulatório, para obtenção de sangue, além de possibilitar a infusão de nutrientes (JACINTO et al., 2014).

Dentro do processo da TIV, a Cateterização Intravenosa Periférica (CIP) é considerada o procedimento invasivo mais utilizado pela equipe de enfermagem em pediatria, constituindo-se como um recurso indispensável na clínica (BATALHA et al., 2010).

No entanto, tal procedimento pode levar ao desenvolvimento de complicações. As complicações decorrentes da TIV são classificadas como locais e sistêmicas. Dentre as complicações locais tem-se o extravasamento (DANSKI et al., 2016).

A Infusion Nurses Society (INS, 2016) define esse evento adverso como a infiltração de um fármaco ou soluções com propriedades vesicantes para tecidos adjacentes.

Os fatores predisponentes associados a extravasamentos em cateteres vasculares periféricos inseridos em crianças e adolescentes hospitalizados não estão bem definidos na literatura nacional e internacional.

Portanto, este estudo tem como objeto de investigação os fatores predisponentes associados a extravasamentos em cateteres vasculares periféricos inseridos em crianças e adolescentes hospitalizados.

A realização deste estudo será relevante do ponto de vista social, pois irá contribuir para a segurança do paciente, diminuindo o número da ocorrência de complicações por extravasamento e os seus efeitos colaterais. Do ponto de vista teórico os dados obtidos contribuirão para o conhecimento dos fatores predisponentes associados a extravasamento em cateteres vasculares periféricos inseridos em crianças e adolescentes hospitalizados, 
prevenindo a ocorrência do mesmo. E do ponto de vista prático, contribuirá para uma prática mais segura e responsável baseada em evidencias cientificas, capacitando os profissionais para prevenir os fatores predisponentes de extravasamento.

\section{METODOLOGIA}

Trata-se de um estudo quantitativo com abordagem transversal. Realizado em um hospital público da cidade de Feira de Santana. Os participantes desse estudo foram crianças e adolescentes hospitalizados na unidade de clínica médica, que apresentaram a necessidade de utilizar a TIV por via periférica. Contou-se com uma amostra de 74 CIP, sendo 43 casos de extravasamento e 31 de termino de terapia intravenosa.

Adotou-se como critério de inclusão: ter idade entre 29 dias a 16 anos incompletos; ter necessidade de terapia intravenosa e medicamentosa por via periférica; ter estabilidade do quadro clínico e estar consciente. E como critério de exclusão: crianças e adolescentes que iniciaram a coleta de dados e desistiram de continuar até o final ou cujos responsáveis solicitaram a interrupção da participação da criança antes do final da coleta; crianças e adolescentes que estiveram clinicamente estáveis no início da pesquisa e ao longo da realização da coleta de dados apresentaram alterações em seu estado de saúde tendendo para o agravamento do mesmo e crianças e adolescentes que foram transferidas para outras unidades de internação de HEC.

Os dados deste estudo foram coletados por meio de fonte primária através da observação da CIP em cada criança selecionada e do prontuário do paciente, por um grupo de coletadores que foram previamente treinados. Assim, após a realização da punção venosa pelos profissionais de enfermagem da unidade em estudo, os dispositivos e a região ao redor do cateter periférico foram observados diariamente, com o objetivo de avaliar as condições da pele, verificando a presença ou a ausência de sinais sugestivos de extravasamento.

Neste estudo foi considerada como variável de desfecho a complicação relacionada à TIV, e como variáveis de exposição às condições sociodemográficos e clínicas das crianças, dados sobre a CIP e TIV utilizada.

Os dados foram tabulados em planilhas eletrônicas e analisados através do programa Statistical Package for the Social Sciences (SPSS), na versão 22.0. Para descrever as variáveis categóricas foram utilizadas distribuições de frequências absolutas e relativas e para as variáveis numéricas as médias e o desvio-padrão. As associações entre as variáveis de exposição e desfecho foram avaliadas através de modelos lineares generalizados, calculandose a razão de prevalência (RP) com intervalo de confiança de $95 \%$. No cruzamento das variáveis, foi utilizado o teste de Qui-quadrado de Pearson e considerados estatisticamente significantes os resultados com o valor de $\mathrm{pp}<0,05$ e para as variáveis com células contendo valores menores que 5, foi utilizado o Teste exato de Fisher, ambos como testes de hipóteses.

O estudo apresentado respeitou os aspectos éticos da Resolução $n^{\circ}$ 466/2012 do Conselho Nacional de Saúde (BRASIL, 2012) e seu mérito ético foi julgado pelo Comitê de Ética em Pesquisa da Universidade Estadual de Feira de Santana, sendo devidamente aprovado através do parecer de número 841.612

\section{RESULTADOS E DISCUSSÃO}

Este estudo apresentou como limitações a incipiente produção do conhecimento sobre extravasamento em crianças e adolescentes na literatura nacional e internacional, ser um estudo de caráter transversal e não termos atingido o tamanho amostral previsto de $118 \mathrm{CIP}$. 
A ocorrência de extravasamento nas CIP realizadas em crianças e adolescentes atingiu uma taxa de $58,1 \%$, estando além da faixa observada na literatura nacional e internacional da área pediátrica, que varia de 4,2\% a 32,2\% (BATALHA et al, 2010; GOMES et al.2011).

Ainda que os resultados encontrados estejam em concordância com a literatura estudada, salienta-se a importância de se oferecer cuidados pautados em evidências cientificas para que complicações na terapia intravenosa sejam prevenidas, pensando-se na segurança e no conforto do paciente pediátrico.

As variáveis demográficas, idade, sexo, raça/cor, condição nutricional e tempo de hospitalização não apresentaram associação estatística, porém verificou-se maior ocorrência de extravasamento em crianças de até seis anos $(63,0 \%)$, do sexo feminino $(60,6 \%)$, desnutridas $(77,7 \%)$, que se auto declararam brancas $(63,6 \%)$, e que permaneceram hospitalizadas por até sete dias $(77,7 \%)$.

No estudo de Negri et. al (2012) os fatores como idade, gênero e cor de pele, também não demonstraram contribuir para o insucesso da CIP. No entanto outros estudos apontam o gênero feminino, pele escura e idade extrema como variáveis que dificultam a obtenção do acesso, e os motivos referidos são decorrentes do menor calibre das veias e dificuldade de avaliar o vaso (GONÇALVES et al 2015; GOMES, A. C. et al., 2011; PEREZ et al., 2015).

Não foram encontradas associações estatísticas entre as variáveis relacionadas à TIV prévia e a ocorrência de extravasamento. No entanto, nota-se uma maior prevalência de extravasamento nas punções em crianças e adolescentes que tem histórico de dificuldade para inserção da CIP (61,0\%), sendo o cateter intravenoso periférico associado ao cateter venoso central mais utilizado previamente $(66,7 \%)$, que tiveram TIV periférica prolongada $(63,2 \%)$, que tiveram antecedentes de complicação $(61,7 \%)$, de infiltração $(62,2 \%)$, e não apresentaram antecedentes de extravasamento $(66,7 \%)$.

No estudo de Negri et al., (2012) encontrou significância estatística ( $p=0,004)$ com história de dificuldade de acesso venoso e o insucesso da CIP, porém o estudo não traz dados sobre a ocorrência de complicação, ainda observou-se que a história prévia de complicações relacionadas ao uso de cateteres aumentou em 2,58 o risco de prevalecer o insucesso na tentativa de punção. Em outro estudo aponta associação estatística entre ter antecedentes de infiltração e flebite e a ocorrência de infiltração ( $p=0,001)$ (JACINTO; AVELAR; PEDREIRA, 2011).

Nas variáveis relacionadas à CIP atual e condição da rede venosa, nota-se que houve associação estatística com a variável local de realização da CIP ( $\mathrm{p}=0,037)$, onde $100 \%$ (6) das Cateterizações Intravenosas Periféricas realizadas no MMII apresentaram extravasamento. Não houve associação em relação às demais variáveis, porém, observou-se uma maior prevalência de extravasamento em crianças e adolescentes com rede venosa não visível (60,7\%), palpável (63,2 \%), tortuosa (70\%), móvel (63,6\%), e superficial (60,4\%). Utilizaram cateter do tipo Poliuretano $(61,3 \%)$, com calibre 24 Gauge $(68,6 \%)$, pelo método indireto de punção $(66,7 \%)$, não tendo o cateter estabilizado $(62,9 \%)$ e que permanecera com a CIP por mais de $72 \mathrm{~h}(58,3 \%)$.

Os locais de inserção do cateter intravenoso periférico devem seguir alguns princípios básicos como, puncionar veias visíveis, relativamente retas e que tenham sofrido o mínimo ou nenhuma manipulação anterior, dar preferência à rede venosa dos membros superiores, evitando os locais de articulação, sendo as veias dos membros inferiores contraindicadas devido ao risco de embolia e tromboflebites (MODES et al., 2011).

Não foram encontradas associações estatísticas entre as variáveis relacionadas à TIV atual e a ocorrência de extravasamento. Observou-se uma maior prevalência de extravasamento em crianças e adolescentes que utilizaram medicamentos irritantes $(60,3 \%)$ e não medicamentos vesicantes $(72,7 \%)$, mas não utilizaram soluções vesicantes $(76,9 \%)$. A prevalência no método misto na administração dos medicamentos $(60 \%)$, a forma de infusão 
da TIV de maneira intermitente e continua associadas $(65,2 \%)$, e o tempo de utilização da terapia medicamentosa de até 7 dias $(59,2 \%)$.

\section{CONSIDERAÇÕES FINAIS}

O presente estudo registrou uma prevalência de $58,1 \%$ de extravasamento, na unidade médica em estudo. Observou-se associação estatística da complicação com o local de

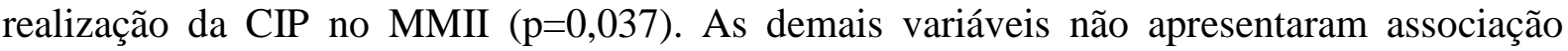
estatística com a ocorrência de extravasamento

Contudo, observou-se maior prevalência de extravasamento em crianças de até seis anos, do sexo feminino, que se auto declararam brancas, que utilizaram a TIV previamente, com cateter venoso periférico, com antecedentes de infiltração, mas que não apresentaram antecedentes de extravasamento, apresentando veias retas, fixas e superficiais, utilizaram cateter $22 \mathrm{G}$, pelo método direto de punção, utilizando duas coberturas, estabilizando o cateter, sendo esse utilizado por mais de 72 horas, que utilizaram medicamentos irritantes, utilizando a TIV por até sete dias.

Propõe-se que sejam realizados novos estudos sobre a ocorrência de extravasamento com crianças e adolescentes, para que seja possível a comparação com os resultados encontrados nesta pesquisa, podendo refuta-los ou confirma-los.

\section{REFERÊNCIAS}

BATALHA, L.M.C. et al. Fixação de cateteres venosos periféricos em crianças: estudo comparativo. Escola Anna Nery (impr.), v.3, n.14, pag. 511-581. Disponível em: <http://www.scielo.br/pdf/ean/v14n3/v14n3a12> . Acessado em 24 maio de 2017.

DANSKI, M.T.R. et al. Incidência de complicações locais e fatores de risco associados ao cateter intravenoso periférico em neonatos. Rev Esc Enferm USP, 2016;50(1):22-28.

INFUSION NURSES SOCIETY BRASIL (INS - BRASIL). Diretrizes práticas para a terapia infusional. São Paulo, 2016.

JACINTO, A.K.L et al. Flebite associada a cateteres intravenosos periféricos em crianças: estudo de fatores predisponentes. Escola Anna Nery Revista de Enfermagem, v.2, n.18, pag. 220-226, 2014.

JACINTO, A. K. L.; AVELAR, A. F. M.; PEDREIRA, M. L. G. Predisposing factors for infiltration in children submitted to peripheral venous catheterization, Journal of Infusion Nursing, Norwood, v. 34, n. 6, p. 391-398, nov/dez., 2011. Disponível em: <https://www.researchgate.net/publication/51815848_Predisposing_Factors_for_Infiltration_in_Childr en_Submitted_to_Peripheral_Venous_Catheterization >. Acesso em: 23 de agosto de 2017.

GOMES, A.V; NASCIMENTO, M. A. L. O processo do cateterismo venoso central em Unidade de Terapia Intensiva Neonatal e Pediátrica. Revista Escola de Enfermagem USP, v.4, n.47, pag.794800, 2013. Disponível em: <http://www.redalyc.org/html/3610/361033327004/>. Acessado em $21 \mathrm{de}$ junho de 2017.

GONÇALVES, A. et al. Caraterização e determinantes do risco de efeito adverso em crianças com cateter venoso periférico: um passo para a melhoria dos cuidados de enfermagem. Revista da UIIP, 2015. Disponível em: <http://ojs.ipsantarem.pt/index.php/REVUIIPS/article/view/93> . Acessado em 19 de maio de 2017.

MODES, P.S.S.A et al. Cuidados de enfermagem nas complicações da punção venosa periférica em recém-nascidos. Revista Rene, v. 12, n. 2, p. 324-332, Fortaleza, 2011. Disponível em: <http://repositorio.ufc.br/ri/handle/riufc/12232> . Acessado em 29 de junho de 2017.

NEGRI, D.C et al. Fatores predisponentes para insucesso da punção intravenosa periférica em crianças. Revista Latino Americana de Enfermagem, v.6, n.20, 2012. Disponível em: <http://www.redalyc.org/html/2814/281424797009/>.. Acessado em 09 de abril de 2017. 